\title{
Review
}

\section{The NFKB Antagonist CDGSH Iron-Sulfur Domain 2 Is a Promising Target for the Treatment of Neurodegenerative Diseases}

\author{
Woon-Man Kung ${ }^{1}$ and Muh-Shi Lin $2,3,4,5, *$ (D) \\ 1 Department of Exercise and Health Promotion, College of Kinesiology and Health, Chinese Culture \\ University, Taipei 11114, Taiwan; nskungwm@yahoo.com.tw \\ 2 Division of Neurosurgery, Department of Surgery, Kuang Tien General Hospital, Taichung 43303, Taiwan \\ 3 Department of Biotechnology and Animal Science, College of Bioresources, National Ilan University, \\ Yilan 26047, Taiwan \\ 4 Department of Biotechnology, College of Medical and Health Care, Hung Kuang University, \\ Taichung 43302, Taiwan \\ 5 Department of Health Business Administration, College of Medical and Health Care, Hung Kuang University, \\ Taichung 43302, Taiwan \\ * Correspondence: neurosurgery2005@yahoo.com.tw; Tel.: +886-4-2665-1900
}

\section{check for}

updates

Citation: Kung, W.-M.; Lin, M.-S The NFkB Antagonist CDGSH Iron-Sulfur Domain 2 Is a Promising Target for the Treatment of Neurodegenerative Diseases. Int. J. Mol. Sci. 2021, 22, 934. https:// doi.org/10.3390/ijms22020934

Received: 26 November 2020

Accepted: 12 January 2021

Published: 19 January 2021

Publisher's Note: MDPI stays neutral with regard to jurisdictional claims in published maps and institutional affiliations.

Copyright: (c) 2021 by the authors. Licensee MDPI, Basel, Switzerland. This article is an open access article distributed under the terms and conditions of the Creative Commons Attribution (CC BY) license (https:// creativecommons.org/licenses/by/ $4.0 /)$.

\begin{abstract}
Proinflammatory response and mitochondrial dysfunction are related to the pathogenesis of neurodegenerative diseases (NDs). Nuclear factor $\kappa \mathrm{B}(\mathrm{NF} \kappa \mathrm{B})$ activation has been shown to exaggerate proinflammation and mitochondrial dysfunction, which underlies NDs. CDGSH iron-sulfur domain 2 (CISD2) has been shown to be associated with peroxisome proliferator-activated receptor- $\beta$ (PPAR$\beta)$ to compete for NFKB and antagonize the two aforementioned NFKB-provoked pathogeneses. Therefore, CISD2-based strategies hold promise in the treatment of NDs. CISD2 protein belongs to the human NEET protein family and is encoded by the CISD2 gene (located at 4q24 in humans). In CISD2, the [2Fe-2S] cluster, through coordinates of 3-cysteine-1-histidine on the CDGSH domain, acts as a homeostasis regulator under environmental stress through the transfer of electrons or iron-sulfur clusters. Here, we have summarized the features of CISD2 in genetics and clinics, briefly outlined the role of CISD2 as a key physiological regulator, and presented modalities to increase CISD2 activity, including biomedical engineering or pharmacological management. Strategies to increase CISD2 activity can be beneficial for the prevention of inflammation and mitochondrial dysfunction, and thus, they can be applied in the management of NDs.
\end{abstract}

Keywords: neurodegenerative disease; CISD2; proinflammatory response; mitochondrial dysfunction; NFKB

\section{Preface}

In the central nervous system (CNS), various complex neurodegenerative processes, including chronic inflammation, continuously occur with natural aging [1].

However, in neurodegenerative diseases (NDs), these processes are much more rapid and pronounced. NDs combined with cognitive or motor deficits are disastrous for patients and contribute a significant burden to the entire society.

Proinflammatory response and mitochondrial dysfunction are driven by activation of nuclear factor kappa-light-chain-enhancer of activated B cells (NFkB) and have been linked to NDs [2-4]. Any novel strategy to suppress NFkB signaling and prevent inflammation and mitochondrial dysfunction may have a therapeutic effect on NDs. This review has focused on CDGSH iron-sulfur domain 2 (CISD2) as a unique zinc finger and iron-sulfur protein and emphasized its protective role against $\mathrm{NF} \kappa \mathrm{B}$ activation. The positive findings regarding the protective role of CISD2 against inflammation and mitochondrial dysfunction support the idea that CISD2 can be a therapeutic target for NDs. 


\section{Inflammation and Mitochondrial Dysfunction Implicated in NDs}

In the pathogenesis of NDs, various microneural abnormalities are involved in structural and functional impairment or loss of CNS neural networks such as enhanced permeability of the blood-brain barrier, damaged axons or myelin sheaths, dysregulated immune activation (innate and adaptive), and neuronal insults $[5,6]$. The abovementioned pathological features commonly underlie NDs such as Alzheimer's disease (AD), Parkinson's disease (PD), multiple sclerosis (MS), and amyotrophic lateral sclerosis (ALS).

Miscellaneous factors, such as genetics, aging, or acquired environmental factors, cause NDs [7]. The well-documented genetic manifestations include the following: (1) autosomal dominant spinocerebellar ataxia type 1 (SCA1): ATXN1 mutation (associated with polyglutamine repeat in ataxin-1) [8]; (2) autosomal dominant early-onset AD: mutations in amyloid precursor protein (chromosome 21), presenilin-1 (chromosome 14), and presenilin2 (chromosome 1) [9]; (3) autosomal dominant early-onset PD: point mutations in SNCA encoding $\alpha$-synuclein [10]; (4) autosomal recessive early-onset PD: mutation in PARK2, PINK1, and PARK7 (encoding parkin, PINK1, and DJ-1 respectively) [11]; early-onset familial forms of ALS: mutation in superoxide dismutase 1 (SOD1) [12]; and (5) autosomal dominant frontotemporal dementia: mutations in the progranulin (PGRN) gene (chromosome 17q21), microtubule-associated protein tau (MAPT) P301L (chromosome 17q21), and chromosome 9 open reading frame 72 (C9orf72) (chromosome 9p21) [13].

The aging process in the CNS has been indicated to precede NDs and is a significant risk factor for NDs. During brain aging, the CNS-resident innate immune cells microglia switch toward detrimental M1 and diminish beneficial M2 phenotypes. Dysfunctional microglia exhibiting resistance to immune adaptive regulation and excessive immunocompetence are highly related to the upregulation of inflammatory genes and, thus, cause persistent neuroinflammation in the CNS [14,15]. Environmental and particularly genetic factors can result in abnormal conformational changes in certain proteins, leading to intracellular and extracellular protein aggregation, which promote neurodegeneration [16]. The abnormally folded/aggregated proteins underlying NDs include hyperphosphorylated tau, A $\beta$-peptide (for AD), $\alpha$-synuclein (for PD), huntingtin (for Huntington disease), and prion (for prion diseases) [17]. Based on this immunocompetent response, aggregation of pattern recognition receptors (PRRs), which are mainly toll-like receptors (TLRs), activate microglia and consequently trigger neuroinflammation [18]. Stimulated M1 phase microglia have been indicated to activate astrocytes directly [19]. Glia-mediated neuroinflammation has been linked to the pathogenesis of various NDs, including AD, PD, and MS [20,21]. These pathological mechanisms, which are consequently detrimental to neuronal survival, involve the generation of various toxic substances, such as reactive oxygen species (ROS), nitrogen species, and proinflammatory mediators [22]. During neuroinflammation, microglia-astrocyte crosstalk facilitates reciprocal modulation of the innate immune defense in the CNS. Stimulated microglia facilitate subsequent astrocytic activation and manipulate the role of astrocytes in neuroprotection or neurotoxicity [23]. Through autocrine feedback, astrocytes inhibit astrocytes [24] or microglial activities and functions [25].

Prolonged neuroinflammation has been demonstrated to result in mitochondrial dysfunction, including altered mitochondrial dynamics, enhanced mitochondrial membrane permeabilization, oxidative phosphorylation, and ROS production [2,26]. As a kind of damage-associated molecular pattern (DAMP), contents of injured mitochondria, such as mitochondrial debris, mitochondrial DNA, and cardiolipin, which are removed by mitophagy, further promote the activation of NACHT, LRR, and PYD domains containing protein 3 (NLRP3) inflammasomes [27]. Consequently, pro-caspase-1 activation and subsequent production of proinflammatory cytokines (IL-1 $\beta$, IL-18) combined with microglial TLR-9 lead to the profound release of proinflammatory mediators [28]. This vicious cascade may expand with the release of more mitochondrial DAMPs, more enhanced inflammasome activation, and more extensive glial neuroinflammation [29]. In summary, pathological mechanisms underlying NDs involve inflammatory responses $[30,31]$ and 
mitochondrial dysfunction [32]. Hence, treatment strategies that exhibit anti-inflammatory and protect mitochondrial function could be potentially beneficial for the management of NDs.

\section{NFKB-Driven Inflammation and Mitochondrial Dysfunction in NDs}

The two critical pathogeneses involved in NDs, inflammation and mitochondrial dysfunction, are provoked by $\mathrm{NF} \kappa \mathrm{B}$. The ND-NFKB axis provides strong rationale for further investigation to modulate NFKB as a therapeutic target in NDs. NFKB serves as a transcription factor in organisms. By combining with a specific nucleotide sequence upstream of the genetic code, $\mathrm{NF} \kappa \mathrm{B}$ can regulate the transcription of genes involved in various vital functions such as inflammation, innate and adaptive immunity, cell differentiation, proliferation, and apoptosis [33].

Structurally, NFKB is a kind of heterodimer or homodimer composed of the following five different subunits: c-Rel, Rel-A (same surname p65), Rel-B, NFkB 1 (same surname p50), and NFkB 2 (same surname p52) [34]. Specifically, p50 and p52 are derived from proteolytic processes of the large precursor proteins p105 and p100, respectively. Each NFKB subunit has been shown to contain the Rel homology domain (RHD) at the $\mathrm{N}$-terminal region [4]. The following functions have been linked to the sequences of RHD: (1) DNA binding; (2) dimerization; (3) binding of inhibitors (IKB proteins) to deactivate nuclear localization signal (NLS) in unstimulated status [35]; and (4) NLS, which guides NFKB translocation from cytoplasm to the nucleus in stimulated status.

The three subunits, c-Rel, p65 (Rel-A), and Rel-B, are characteristic of a transactivation domain (TAD) [36], which serves as an activation zone through the combination with various kinds of transcriptional factors, such as transcriptional co-activators cAMPresponse-element-binding-protein-binding protein (CBP). Through the connection of TADs with transcriptional factors, transcription reactions can occur.

As the well-documented pathway of canonical NFKB activation, various PRRs act as environmental stimuli to immune cells (such as pathogen-associated molecular patterns, or DAMPs) to activate the IкB kinase (IKK) complex. IKK-associated cytoplasmic signals result in IKB (an inhibitor of $\kappa B$ ) phosphorylation and subsequent degradative ubiquitination. As described previously, IKB degradation leads to unmasking of NLPs in p65/p50 or p50/c-Rel dimers, nuclear translocation of $\mathrm{NF} \kappa \mathrm{B}$ from cytoplasm, and consequent proinflammatory signal transduction [33]. Specifically, the IKK complex consists of the following three subunits: IKK- $\alpha$, IKK- $\beta$, and IKK- $\gamma$. IKK- $\alpha$ and IKK- $\beta$ serve as catalytic components of the IKK complex. In addition, IKK- $\gamma$ has been shown to contain a C-terminal zinc finger domain of the C2HC type [37]. The zinc finger of IKK- $\gamma$ potentially upregulates or downregulates the activity of IKK complexes by binding to upstream proteins. Ubiquitin recognition and the binding of IKK- $\gamma$ zinc fingers are related to the regulation of $\mathrm{NFKB}$, and thus, IKK- $\gamma$ is considered a NFKB essential modulator (NEMO).

In addition to the cytoplasm, $N F K B$ is detectable in mitochondria [4]. It regulates mitochondrial function [3] and modulates the activities of NLRP3 inflammasomes in the endoplasmic reticulum (ER) and mitochondria-associated ER membranes (MAMs) [38]. In mitochondria, $\mathrm{NF} \kappa \mathrm{B}$ has been verified to be involved in the regulation of the following mitochondrial functions: mitochondrial dynamics, activities of respiratory complexes for electron transport chain, release of cytochrome C, and cellular apoptosis. Furthermore, an activated form of NLRP3 inflammasomes has been presented to migrate to the MAM from the ER membrane [38] after receiving priming signals, such as ligands of TLRs, mitochondrial ROS, and cytokines. Moreover, after activation of NLRP3 inflammasomes, advanced cytokine loops (IL-1 $\beta$, IL-18) are subsequently formed, which can then stimulate the generation of proinflammatory cascades and eventually establish a vicious cycle between inflammation and mitochondrial dysfunction. NFKB-regulated NLRP3 inflammasomes activation involves the following critical organelles significant to cellular function: mitochondria, ER membranes, and MAMs [39]. 


\section{The Evolutionary Conserved NEET Family Member CISD2 Regulates Important Homeostatic CNS Functions such as $\mathrm{pH}$ and Oxidation State}

Cytogenetically, CISD2 is located at the long arm of chromosome 4 at position 24 (4q24) in humans [40]. The synonyms of CISD2 documented in the literature are as follows: nutrient-deprivation autophagy factor-1 (NAF-1), ER intermembrane small protein (Eris), mitoNEET related 1 (Miner 1), Wolfram syndrome 2 (WFS2), zinc finger, CDGSHtype domain 2 (ZCD2), and nervous system overexpressed protein 70 (Noxp70). Three small exons with an average size of $24 \mathrm{~kb}$ are included in the gene transcript of CISD2. Transcript accession identifiers of CISD2 are as follows: NM_001008388.4 (NCBI databases), ENST00000273986, ENST00000503643, and ENST00000574446 (Ensembl databases) [41].

CISD2 encodes the CISD2 protein, which is the second member of the three members of the human NEET family (CISD1-3); hence, it is called CISD2. In the NEET protein family, the following amino acid sequence commonly appears in the C-terminal of each family member Asn-Glu-Glu-Thr (NEET) [42]. Hence, the protein family is named NEET. Moreover, all the members of the NEET protein family share the following feature: (1) the sequence motif, CDGSH (39 amino acid stretch) [43]; (2) the combination of CDGSH domain with the [2Fe-2S] cluster through coordinates of 3-cysteine (Cys)-1-histidine (His) on the CDGSH motif [44]. Thus, the NEET protein family is characterized by the unique combination of 3Cys-1His CDGSH 2Fe-2S, [C-X-C-X2-(S/T)-X3-P-X-C-D-G-(S/A/T)-H] [45,46]. The CDGSH domain can be detected in archaea, bacteria, plants, and humans, which provides evidence of high evolutionary conservation [47].

In humans, three members of the NEET protein family, CISD1-3 [48-50], have been well documented. Due to the specific feature (CDGSH iron-sulfur domain), the three human NEET proteins are named CISD1-3 (based on the first letter of each word). The morphological differences of the NEET protein family can be divided into the following two categories [46]: (1) homodimer with each monomer with one CDGSH domain (Class I NEET proteins, e.g., CISD1 and CISD2); and (2) monomeric proteins with two CDGSH domains (Class II NEET proteins, e.g., bacterial mitochondrial inner NEET protein [MiNT] and CISD3).

On the coordination of (Cys)3(His)1-[2Fe-2S] cluster, the histidine ligand (His114) of CISD2 protein combines with the [2Fe-2S] cluster via N $\delta$ [51,52]. Significantly, His114 influences the [2Fe-2S] cluster binding affinity according to the redox state of the cluster or environmental $\mathrm{pH}$ value. As the $\mathrm{pH}$ declines to 7 , protonation of the histidine ligand can be detected in CISD2 proteins, which leads to the transfer of [2Fe-2S] cluster, i.e., cluster transfer [53]. The cluster transfer of NEET proteins is accelerated by reducing the $\mathrm{pH}$ levels [54].

In addition to the $\mathrm{pH}$ effect, the extent of the oxidation state of the [2Fe-2S] cluster influences NEET-cluster lability. The [2Fe-2S] cluster of NEET proteins has been shown to be structurally stable in the reduced state [55]. Under environmental stress, vital iron-sulfur proteins may be enzymatically inactivated, and the function is impaired due to the loss of [Fe-S] clusters caused by damage; for example, an apo-protein receptor involving in iron homeostasis, an inactive form of ion regulatory protein 1 (IRP1), and cytosolic aconitase [56]. The impaired cluster function of these damaged proteins can be rescued by NEET proteins as a potential reducing agent. The mitochondrial antioxidant effect of NEET proteins can be achieved by electron transfer from the cluster [57]. Specifically, the alternation in the redox status of [Fe-S] clusters contributes to cluster transfer [58-60]. [Fe-S] clusters change from a reduced (rested) to an oxidation (activated) state and subsequently result in cluster transfer to the apo-protein receptors. After the donation of clusters from NEET proteins, these injured proteins can potentially be repaired. With a protective effect commonly shared by the NEET family, the [2Fe-2S] cluster in CISD2 acts as a homeostasis maintainer under environmental stress through the transfer of electrons or iron-sulfur clusters.

Structurally, the CISD2 protein possesses a common frame with the NEET protein, folds into a homodimeric structure in a unique form, and generates the so-called NEET fold (two-fold pseudo symmetric backbone). The two specific domains, $\beta$-cap (comprising 
three long $\beta$-strands) and cluster binding (including C-terminal [2Fe-2S] CDGSH motif and $\alpha$-helix structure), constitute the main structure of CISD2 in each monomer $[47,53]$. In general, a hydrophobic core has been detected in the $\beta$-cap domain. The cluster binding domain is hydrophilic and charged. These characteristics are related to protein folding and the combinations with specific molecules through the domains [61].

Most of the NEET conformation, including CISD, is situated in the cytoplasm, and these parts of the CISD2 protein form the cytosolic domain. In addition to these functional parts of NEET folds, CISD2 protein has transmembrane helices at their N-terminal region and in-organelle domain [47]. Each monomer of homodimeric CISD2 protein anchors to the outer membrane of mitochondria (OMM) through the transmembrane helix [53]. The other two subcellular locations reported in the literature include the ER and MAMs. Specifically, CISD2 has been proven to be an integral membrane protein with a C-terminal EGFP tag on ER membrane [50] and is abundant in MAMs [62,63].

The CDGSH motif of NEET fold in the CISD2 protein has been indicated to be a CDGSH-type zinc finger [45,64] with the characteristic of a CysCysCysHis finger [65] (serves as Cys3/His1 type, C3H1, among nine subgroups of all zinc finger proteins [66]). Although annotated as zinc fingers, the $\mathrm{C} 3 \mathrm{H} 1$ ligand set coordinates with the [2Fe-2S] cluster instead of zinc in CISD2. It has been postulated that insults from oxidative stress or ROS formation may destroy the zinc finger structure, especially the coordinates of Cys and His [67]. Redox properties of [2Fe-2S] clusters is associated with the activity of zinc finger motifs of CISD2 [68].

\section{Physiological Function of CISD2}

In ER or MAMs, CISD2 mediates pivotal functions, including lipid synthesis and protein folding [64]. CISD2 can act as a transport conduit for redox calcium between the ER, MAMs, mitochondria, and the cytosol. As such, CISD2 can regulate redox reactions, which is critical for mitochondrial modulation relevant to NDs. [Fe-S] cluster transfer from CISD1 to CISD2 has been shown to achieve effective transport of labile iron from mitochondria to the cytosol to prevent mitochondrial Fe/ROS/Fe-S dyshomeostasis [69]. CISD2 mutation potential leads to mitochondrial iron overload and ROS toxicity [70]. Moreover, protection against apoptosis-related mitochondrial dysfunction of CISD2 in combination with BCL2 has been proven to be ineffective under the circumstances of CISD2 absence from the [2Fe-2S] cluster [71].

CISD2 is interesting for providing the following inhibitory functions to protect cells from damage:

(1) Calcium excitotoxicity. CISD2 has been shown to depress excitotoxic $\mathrm{Ca}^{2+}$ increase at the ER through the binding of CISD2 to BCL2 along with the inositol 1,4,5triphosphate (IP3) receptor (a kind of calcium channel in the ER membrane) [72,73]. CISD2 deficiency can trigger an increase in ER and cytoplasmic $\mathrm{Ca}^{2+}$ levels in CISD2 knockout mice compared with wild-type mice [72]. Furthermore, results obtained from CISD2 knockout mice showed that CISD2 along with GTPase of immuneassociated protein 5 (Gimap5) in MAMs decreased cytosolic $\mathrm{Ca}^{2+}$ surge and enhanced mitochondrial $\mathrm{Ca}^{2+}$ uptake [63].

(2) Apoptosis. CISD2 along with the IP3 receptor has been shown to connect with a combination of Bcl-2-Beclin-1 complex in the ER membrane. Bcl-2 exerts its antiapoptotic effect via antagonism of Beclin-1. As a Bcl-2-interacting protein, CISD2 functions as an autophagy regulator. The binding of CISD2 to Bcl-2 regulates Bcl-2 to antagonize autophagy/apoptosis in response to stress [71,74]. CISD2 enhances Bcl-2-Beclin-1 interaction and prevents the potential apoptotic cellular damage. When CISD2 is attenuated, the interaction between Bcl-2 and Beclin-1 is greatly reduced, and autophagy is triggered. As mentioned earlier, the effect generated by the combination of CISD2 and Bcl-2 is invalid when CISD2 loses the [2Fe-2S] cluster [71].

(3) OMM breakdown and subsequent mitochondrial abnormality. The most prominent study on mitochondrial CISD2 function from CISD2 knockout mice was published 
by a group of Taiwanese neuroscientists headed by Ting-Fen Tsai in 2009 [75]. As a part of OMMs, CISD2 proteins play a vital role in the maintenance of mitochondrial function. Studies on CISD2 $^{-/-}$mice have demonstrated that CISD2 knockout induces mitochondrial degeneration, autophagy, and consequent enhancement of the aging process. As a kind of ER/mitochondria-related disease, WFS2, a subtype among WFS (featured in diabetes insipidus, diabetes, optic atrophy, and deafness [DIDMOAD]), has been linked to the recessive mutation of CISD2. WFS2 may clinically manifest as diabetes mellitus, optic atrophy, and a bleeding tendency [76].

6. CISD2 as a NFKB Antagonist against Inflammation and Mitochondrial Dysfunction: A Promising Target for NDs

The research team has addressed the vital role of CISD2 protein in preventing inflammation and mitochondrial dysfunction. A CISD2 knockdown model using siCISD2 in immune and non-immune cells was applied to verify the potential function of CISD2. The following findings were revealed: (1) enhanced inflammatory responses (elevated production of iNOS and chemokine regulated on activation, normal $\mathrm{T}$ cell expressed and secreted [RANTES]), extensive mitochondrial dysfunction (including decreased mitochondrial membrane potential DeltaPsi(m) and elevated ROS release), promoted apoptosis, and decreased cell viability in neuron-like cells, SH-SY5Y [77,78]; and (2) augmented inflammatory reaction, as indicated in enhanced M1 microglia polarization (increased expression of TNF- $\alpha$, IL-1 $\beta$, iNOS, and COX2), attenuated M2 microglia phenotype (decreased expression of Arg-1, Ym1, and IL-10), and promoted apoptosis in EOC microglial cells [79].

Moreover, the anti-inflammatory effect of CISD2 has been shown to antagonize the activation of NFKB. Enhanced activity of NFKB p65 subunit DNA binding and subsequent nuclear translocation of NFKB p65 have been confirmed in siCISD2-transfected EOC microglial cells [79]. CISD2 inhibits NFKB signaling by acting upstream of the peroxisome proliferator-activated receptor (PPAR)- $\beta$ (synonym: PPAR- $\delta$ ), which is due to the decrease of PPAR- $\beta$ expression shown in SH-SY5Y with CISD2 knockdown [80]. The beneficial effect of PPAR- $\beta$ has been demonstrated to prevent I $\kappa B$ degradation and consequent NF $\kappa B$ activation $[81,82]$. It has been established that the anti-inflammatory effect of CISD2 is through the upstream regulation of PPAR- $\beta / \mathrm{NFKB}$ signaling.

\section{CISD2 Attenuation on Neural Pathology}

As a regulator of NFKB activation, CISD2 expression levels have been demonstrated to decrease during neurological pathology. CISD2 attenuation leads to reduced suppression against NFKB activation. Therefore, the proinflammatory response and mitochondrial dysfunction caused by NFKB are manifested in the cytoplasm and mitochondria. It is worth noting that the inflammation and mitochondrial dysfunction caused by CISD2 decline are widely involved in neurodegenerative states and neurotrauma. Specifically, CISD2 expression was found to be decreased in the brain and spinal cord of aging mice compared to that in the brain and spinal cord of young mice. A reduction of CISD2 expression and enhanced production of proinflammatory mediators were confirmed in the group of 35 days in vitro (DIV) long-term primary culture of astrocyte compared to those in the group of 7 DIV cells [78]. Similar effects for age-dependent CISD2 decline have been reported in mouse tissues of the brain, skin, and skeletal muscles [83]. In addition, cell injured models of LPS challenge or animal models of spinal cord hemisection have been shown to reduce the expression levels of CISD2 in primary astrocytes [77], ALT astrocytes [80], and mice [77,80], respectively. Therefore, CISD2 decline can be observed in neural pathology involving either the neurodegenerative process or neurological trauma. As mentioned above, detailed pathological effects of inflammation and mitochondrial dysfunction caused by insult-attenuated CISD2 expression are well described in cell experiments with CISD2 knockdown. 


\section{CISD2-Elevating Strategy as the Potential Future Therapy in NDs}

NFKB-evoked critical mechanisms, inflammation and mitochondrial dysfunction, underlie the pathogenesis of NDs. The protective effect of CISD2 has been established to inhibit the above two pathological mechanisms and NFKB activation. Hence, CISD2 is considered as a potential therapeutic target for NDs. As discussed above, CISD2 expression is reduced under the circumstances of CNS degenerative status or injuries. Therapeutic strategies that focus on how to increase the expression level of CISD2 are promising for the future treatment of NDs. Below, we address the previously reported CISD2-elevating strategies.

\section{Strategy 1. Experimental Over-Expression}

When CISD2 is overexpressed in AD mice, reduced neuron loss, $\beta$-amyloid-induced mitochondrial dysfunction, and decreased immunofluorescence of Iba1 (ionized calciumbinding adapter molecule 1) and GFAP have been reported in the hippocampus [84].

Strategy 2. Experimental Cryogen Spray Cooling

Cryogen spray cooling has been used for the management of spinal cord injury, showing protection against injury-induced CISD2 decline and astrocyte-mediated neuroinflammation in a rat sustained spinal cord hemisection [85].

\section{Strategy 3. Curcumin}

Studies published in the literature support that treatment strategies of raising CISD2 have a protective effect on CNS injuries and diseases. Aged mice (104 weeks) and 35 DIV primary cultured astrocytes have shown to have decreased CISD2 expression levels; such aging-driven CISD2 loss can be attenuated after curcumin treatment in vivo and in vitro [78]. Similar effects have been seen in injury experimental models. Curcumin has been demonstrated to reduce CISD2 loss in mice with spinal cord injury and LPS-challenged astrocytes [77].

Strategy 4. Momordica Charantia Linn. var. Abbreviata Ser. (WBM)

A similar pattern of reduction in CISD2 protein after SCI and in LPS-stimulated astrocytic cell lines (ALT) also improves by the treatment of WBM. The decrease in glial activation and proinflammatory cascades is also accompanied by an increase in the levels of CISD2 protein after WBM treatment [80].

As mentioned above, CISD2 can be pathologically attenuated in either neurodegenerative or neurotraumatic status and cause NFkB-provoked inflammation and mitochondrial dysfunction. The proposed strategies 1-4 exhibit CISD2-preserving effects, which contribute to the prevention of inflammation and mitochondrial dysfunction. CISD2 augmentation can help overcome the detrimental effects of NFKB signaling and can be considered a potential therapeutic target for CNS injury or disease. In summary, novel strategies involving biomedical engineering or pharmacological novel agents that help CISD2 elevation can be suggested for the management of NDs.

\section{Conclusions}

With the increase in the aging population worldwide, the number of people affected by NDs may rise rapidly. This significant issue needs to be addressed. NDs commonly cause physical impairment and functional disability among the elderly population worldwide, increasing the burden on society. Several factors susceptible to this disease category include genetics, aging, and the environment, which may lead to abnormal aggregation of misfolded proteins in the CNS (Figure 1) and further trigger neuroinflammation, mitochondrial dysfunction, neuronal loss, and eventual neurological deficits. 


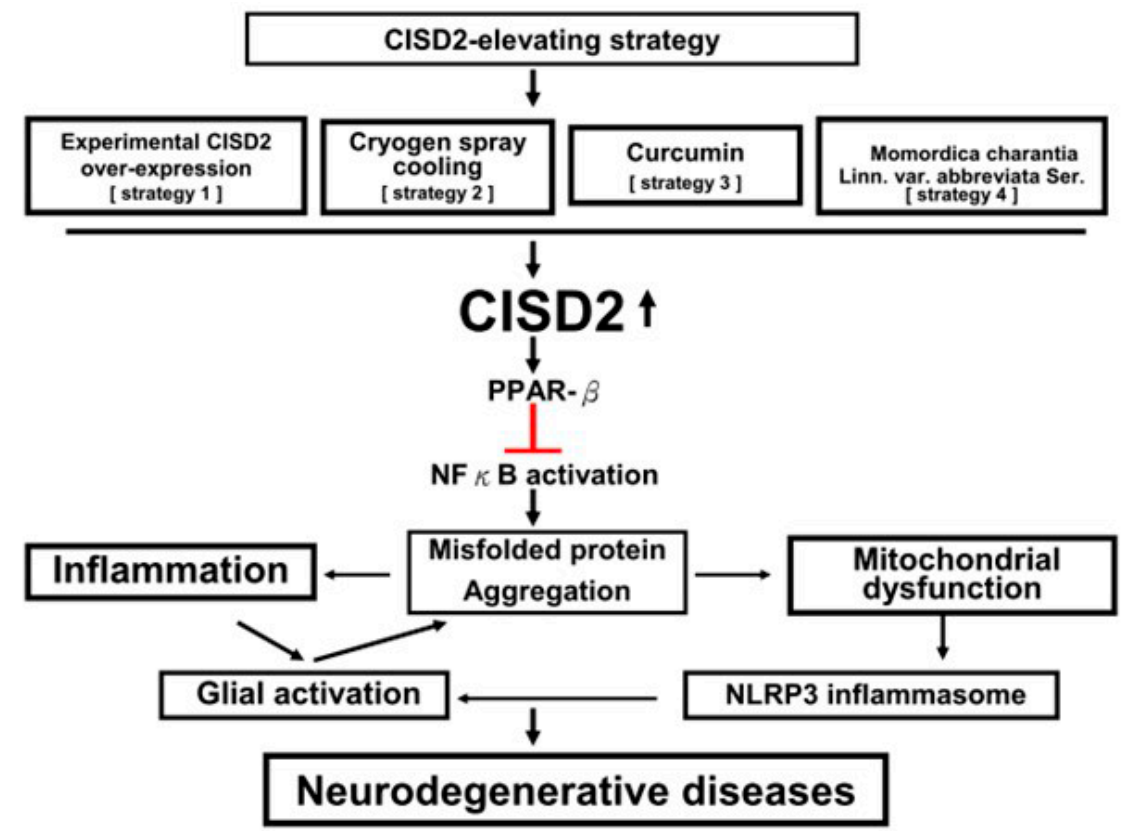

Figure 1. Diagram of the benefit of CISD2-elevating strategies in neurodegenerative diseases (NDs). NFkB-provoked inflammation and mitochondrial dysfunction underlie the pathogenesis of NDs. Accumulation of misfolded proteins in the central nervous system (CNS) induces neuroinflammation by triggering the activation of microglia, astrocytes, and glia-secreted proinflammatory cytokines/chemokines. Advanced neuroinflammation consequently leads to mitochondrial dysfunction and the production of mitochondrial damage-associated molecular patterns (DAMP), which promote the activation of NLRP3 inflammasome and subsequently exaggerate neuroinflammation.

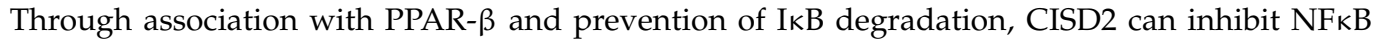
activation (red bar). Therapeutic strategies that upregulate CISD2 have the potential to attenuate the PPAR- $\beta / \mathrm{I} \kappa \mathrm{B} / \mathrm{NF} \kappa \mathrm{B}$ signaling pathway and mitigate NFKB-provoked inflammation and mitochondrial dysfunction implicated in NDs.

As shown in this review article, the function of CISD2 is not limited to calcium metabolism, anti-apoptosis, and longevity. CISD2 functions as a family of NEET proteins along with zinc finger proteins possessing the [2Fe-2S] cluster. These properties enable the protein to regulate vital proteins, such as $\mathrm{Bcl} 2, \mathrm{PPAR}-\beta, \mathrm{NF} \kappa \mathrm{B}$, and CISD1. Through cluster reception/donation, CISD2 combined with $\mathrm{Bcl} 2$ and CISD1 can regulate the key physiological mechanisms of apoptosis-related mitochondrial function/mitochondrial inflammasome activation and mitochondrial Fe/ROS/Fe-S homeostasis, respectively. Upstream modulation of PPAR- $\beta / \mathrm{NF} \kappa \mathrm{B}$ proinflammatory signaling of CISD2 provides a rationale for the management of the two NFKB-provoked pathological mechanisms (neuroinflammation and mitochondrial dysfunction), which underlie NDs (Figure 1). Novel strategies targeted to elevate CISD2 are expected to be used in the management of NDs.

Author Contributions: W.-M.K.: original draft, investigation and formal analysis; M.-S.L.: conceptualization, supervision, formal analysis and writing-review and editing. All authors have read and agreed to the published version of the manuscript.

Funding: This research received no external funding.

Conflicts of Interest: The authors declare no conflict of interest. 


\section{References}

1. Sanada, F.; Taniyama, Y.; Muratsu, J.; Otsu, R.; Shimizu, H.; Rakugi, H.; Morishita, R. Source of Chronic Inflammation in Aging. Front. Cardiovasc. Med. 2018, 5, 12. [CrossRef] [PubMed]

2. Missiroli, S.; Genovese, I.; Perrone, M.; Vezzani, B.; Vitto, V.A.M.; Giorgi, C. The Role of Mitochondria in Inflammation: From Cancer to Neurodegenerative Disorders. J. Clin. Med. 2020, 9, 740. [CrossRef] [PubMed]

3. Laforge, M.; Rodrigues, V.; Silvestre, R.; Gautier, C.; Weil, R.; Corti, O.; Estaquier, J. NF-kB pathway controls mitochondrial dynamics. Cell Death Differ. 2016, 23, 89-98. [CrossRef] [PubMed]

4. Albensi, B.C. What Is Nuclear Factor Kappa B (NF-kB) Doing in and to the Mitochondrion? Front. Cell Dev. Biol. 2019, 7 , 154. [CrossRef]

5. Datta, G.; Colasanti, A.; Rabiner, E.A.; Gunn, R.N.; Malik, O.; Ciccarelli, O.; Nicholas, R.; Van, V.E.; Van, H.W.; Searle, G.; et al. Neuroinflammation and its relationship to changes in brain volume and white matter lesions in multiple sclerosis. Brain 2017, 140, 2927-2938. [CrossRef]

6. Chen, W.W.; Zhang, X.; Huang, W.J. Role of neuroinflammation in neurodegenerative diseases (Review). Mol. Med. Rep. 2016, 13, 3391-3396. [CrossRef]

7. Wyss-Coray, T. Ageing, neurodegeneration and brain rejuvenation. Nature 2016, 539, 180-186. [CrossRef]

8. Perez Ortiz, J.M.; Orr, H.T. Spinocerebellar Ataxia Type 1: Molecular Mechanisms of Neurodegeneration and Preclinical Studies. Adv. Exp. Med. Biol. 2018, 1049, 135-145.

9. Jia, L.; Fu, Y.; Shen, L.; Zhang, H.; Zhu, M.; Qiu, Q.; Wang, Q.; Yan, X.; Kong, C.; Hao, J.; et al. PSEN1, PSEN2, and APP mutations in 404 Chinese pedigrees with familial Alzheimer's disease. Alzheimer's Dement. 2020, 16, 178-191. [CrossRef]

10. Olgiati, S.; Thomas, A.; Quadri, M.; Breedveld, G.J.; Graafland, J.; Eussen, H.; Douben, H.; de Klein, A.; Onofrj, M.; Bonifati, V. Early-onset parkinsonism caused by alpha-synuclein gene triplication: Clinical and genetic findings in a novel family. Parkinsonism Relat. Disord. 2015, 21, 981-986. [CrossRef]

11. Hauser, D.N.; Mamais, A.; Conti, M.M.; Primiani, C.T.; Kumaran, R.; Dillman, A.A.; Langston, R.G.; Beilina, A.; Garcia, J.H.; Diaz-Ruiz, A.; et al. Hexokinases link DJ-1 to the PINK1/parkin pathway. Mol. Neurodegener. 2017, 12, 70. [CrossRef] [PubMed]

12. Abati, E.; Bresolin, N.; Comi, G.; Corti, S. Silence superoxide dismutase 1 (SOD1): A promising therapeutic target for amyotrophic lateral sclerosis (ALS). Expert Opin. Ther. Targets. 2020, 24, 295-310. [CrossRef] [PubMed]

13. Greaves, C.V.; Rohrer, J.D. An update on genetic frontotemporal dementia. J. Neurol. 2019, 266, 2075-2086. [CrossRef]

14. Olmedillas Del, M.M.; Asavapanumas, N.; Uzcátegui, N.L.; Garaschuk, O. Healthy Brain Aging Modifies Microglial Calcium Signaling In Vivo. Int. J. Mol. Sci. 2019, 20, 589. [CrossRef]

15. Matt, S.M.; Johnson, R.W. Neuro-immune dysfunction during brain aging: New insights in microglial cell regulation. Curr. Opin. Pharmacol. 2016, 26, 96-101. [CrossRef]

16. Tcw, J.; Goate, A.M. Genetics of $\beta$-Amyloid Precursor Protein in Alzheimer's Disease. Cold Spring Harb. Perspect. Med. 2017, 7 , a024539. [CrossRef]

17. Ma, B.; Zhao, J.; Nussinov, R. Conformational selection in amyloid-based immunotherapy: Survey of crystal structures of antibody-amyloid complexes. Biochim. Biophys. Acta (BBA) Gen. Subj. 2016, 1860, 2672-2681. [CrossRef]

18. Venegas, C.; Heneka, M.T. Danger-associated molecular patterns in Alzheimer's disease. J. Leukoc. Biol. 2017, 101, 87-98. [CrossRef]

19. Liu, L.R.; Liu, J.C.; Bao, J.S.; Bai, Q.Q.; Wang, G.Q. Interaction of Microglia and Astrocytes in the Neurovascular Unit. Front. Immunol. 2020, 11, 1024. [CrossRef]

20. Ransohoff, R.M. How neuroinflammation contributes to neurodegeneration. Science 2016, 353, 777-783. [CrossRef]

21. Chitnis, T.; Weiner, H.L. CNS inflammation and neurodegeneration. J. Clin. Investig. 2017, 127, 3577-3587. [CrossRef] [PubMed]

22. McElroy, P.B.; Liang, L.P.; Day, B.J.; Patel, M. Scavenging reactive oxygen species inhibits status epilepticus-induced neuroinflammation. Exp. Neurol. 2017, 298, 13-22. [CrossRef]

23. Norden, D.M.; Muccigrosso, M.M.; Godbout, J.P. Microglial priming and enhanced reactivity to secondary insult in aging, and traumatic CNS injury, and neurodegenerative disease. Neuropharmacology 2015, 96, 29-41. [CrossRef] [PubMed]

24. Guo, Y.S.; Liang, P.Z.; Lu, S.Z.; Chen, R.; Yin, Y.Q.; Zhou, J.W. Extracellular ab-crystallin modulates the inflammatory responses. Biochem. Biophys. Res. Commun. 2019, 508, 282-288. [CrossRef]

25. Jha, M.K.; Jo, M.; Kim, J.H.; Suk, K. Microglia-Astrocyte Crosstalk: An Intimate Molecular Conversation. Neuroscientist 2019, 25, 227-240. [CrossRef]

26. Zhou, D.; Jiang, Y. Sirtuin 3 attenuates neuroinflammation-induced apoptosis in BV-2 microglia. Aging 2019, 11, 9075-9089. [CrossRef]

27. Yoo, S.M.; Park, J.; Kim, S.H.; Jung, Y.K. Emerging perspectives on mitochondrial dysfunction and inflammation in Alzheimer's disease. BMB Rep. 2020, 53, 35-46. [CrossRef]

28. Santoni, G.; Cardinali, C.; Morelli, M.B.; Santoni, M.; Nabissi, M.; Amantini, C. Danger- and pathogen-associated molecular patterns recognition by pattern-recognition receptors and ion channels of the transient receptor potential family triggers the inflammasome activation in immune cells and sensory neurons. J. Neuroinflamm. 2015, 12, 21. [CrossRef] [PubMed]

29. Van Horssen, J.; van Schaik, P.; Witte, M. Inflammation and mitochondrial dysfunction: A vicious circle in neurodegenerative disorders? Neurosci. Lett. 2019, 710, 132931. [CrossRef]

30. Hickman, S.; Izzy, S.; Sen, P.; Morsett, L.; El, K.J. Microglia in neurodegeneration. Nat. Neurosci. 2018, 21, 1359-1369. [CrossRef] 
31. Ravari, A.; Mirzaei, T.; Kennedy, D.; Kazemi, A.M. Chronoinflammaging in Alzheimer; A systematic review on the roles of toll like receptor 2. Life Sci. 2017, 171, 16-20. [CrossRef]

32. Sun, N.; Youle, R.J.; Finkel, T. The Mitochondrial Basis of Aging. Mol. Cell 2016, 61, 654-666. [CrossRef] [PubMed]

33. Liu, T.; Zhang, L.; Joo, D.; Sun, S.C. NF-kB signaling in inflammation. Signal Transduct. Target. Ther. 2017, 2, 17023. [CrossRef]

34. Sun, S.C.; Chang, J.H.; Jin, J. Regulation of nuclear factor-kB in autoimmunity. Trends Immunol. 2013, 34, 282-289. [CrossRef]

35. Latimer, M.; Ernst, M.K.; Dunn, L.L.; Drutskaya, M.; Rice, N.R. The N-terminal domain of IkappaB alpha masks the nuclear localization signal(s) of p50 and c-Rel homodimers. Mol. Cell Biol. 1998, 18, 2640-2649. [CrossRef] [PubMed]

36. Hoffmann, A.; Natoli, G.; Ghosh, G. Transcriptional regulation via the NF-kappaB signaling module. Oncogene 2006, 25, 6706-6716. [CrossRef]

37. Shifera, A.S. The zinc finger domain of IKK gamma (NEMO) protein in health and disease. J. Cell. Mol. Med. 2010, 14, 2404-2414. [CrossRef]

38. Missiroli, S.; Patergnani, S.; Caroccia, N.; Pedriali, G.; Perrone, M.; Previati, M.; Wieckowski, M.R.; Giorgi, C. Mitochondriaassociated membranes (MAMs) and inflammation. Cell Death Dis. 2018, 9, 329. [CrossRef]

39. Zhou, Y.; Tong, Z.; Jiang, S.; Zheng, W.; Zhao, J.; Zhou, X. The Roles of Endoplasmic Reticulum in NLRP3 Inflammasome Activation. Cells 2020, 9, 1219. [CrossRef]

40. Amr, S.; Heisey, C.; Zhang, M.; Xia, X.J.; Shows, K.H.; Ajlouni, K.; Pandya, A.; Satin, L.S.; El-Shanti, H.; Shiang, R. A homozygous mutation in a novel zinc-finger protein, ERIS, is responsible for Wolfram syndrome 2. Am. J. Hum. Genet. 2007, 81, 673-683. [CrossRef]

41. Cattaneo, M.; La, S.L.; Rondinelli, M.; Errichiello, E.; Zuffardi, O.; Puca, A.A.; Genovese, S.; Ceriello, A. A donor splice site mutation in CISD2 generates multiple truncated, non-functional isoforms in Wolfram syndrome type 2 patients. BMC Med. Genet. 2017, 18, 147. [CrossRef]

42. Moreno-Navarrete, J.M.; Moreno, M.; Ortega, F.; Sabater, M.; Xifra, G.; Ricart, W.; Fernández-Real, J.M. CISD1 in association with obesity-associated dysfunctional adipogenesis in human visceral adipose tissue. Obesity 2016, 24, 139-147. [CrossRef] [PubMed]

43. Lin, J.; Zhang, L.; Lai, S.; Ye, K. Structure and molecular evolution of CDGSH iron-sulfur domains. PLoS ONE 2011, 6, e24790. [CrossRef] [PubMed]

44. Pesce, L.; Calandrini, V.; Marjault, H.B.; Lipper, C.H.; Rossetti, G.; Mittler, R.; Jennings, P.A.; Bauer, A.; Nechushtai, R.; Carloni, P. Molecular Dynamics Simulations of the [2Fe-2S] Cluster-Binding Domain of NEET Proteins Reveal Key Molecular Determinants That Induce Their Cluster Transfer/Release. J. Phys. Chem. B 2017, 121, 10648-10656. [CrossRef]

45. Wiley, S.E.; Murphy, A.N.; Ross, S.A.; van der Geer, P.; Dixon, J.E. MitoNEET is an iron-containing outer mitochondrial membrane protein that regulates oxidative capacity. Proc. Natl. Acad. Sci. USA 2007, 104, 5318-5323. [CrossRef]

46. Inupakutika, M.A.; Sengupta, S.; Nechushtai, R.; Jennings, P.A.; Onuchic, J.N.; Azad, R.K.; Padilla, P.; Mittler, R. Phylogenetic analysis of eukaryotic NEET proteins uncovers a link between a key gene duplication event and the evolution of vertebrates. Sci. Rep. 2017, 7, 42571. [CrossRef]

47. Karmi, O.; Marjault, H.B.; Pesce, L.; Carloni, P.; Onuchic, J.N.; Jennings, P.A.; Mittler, R.; Nechushtai, R. The unique fold and lability of the [2Fe-2S] clusters of NEET proteins mediate their key functions in health and disease. J. Biol. Inorg. Chem. 2018, 23, 599-612. [CrossRef]

48. Kusminski, C.M.; Holland, W.L.; Sun, K.; Park, J.; Spurgin, S.B.; Lin, Y.; Askew, G.R.; Simcox, J.A.; McClain, D.A.; Li, C.; et al. MitoNEET-driven alterations in adipocyte mitochondrial activity reveal a crucial adaptive process that preserves insulin sensitivity in obesity. Nat. Med. 2012, 18, 1539-1549. [CrossRef]

49. Nechushtai, R.; Conlan, A.R.; Harir, Y.; Song, L.; Yogev, O.; Eisenberg-Domovich, Y.; Livnah, O.; Michaeli, D.; Rosen, R.; Ma, V.; et al. Characterization of Arabidopsis NEET reveals an ancient role for NEET proteins in iron metabolism. Plant Cell 2012, 24, 2139-2154. [CrossRef]

50. Wiley, S.E.; Andreyev, A.Y.; Divakaruni, A.S.; Karisch, R.; Perkins, G.; Wall, E.A.; van der Geer, P.; Chen, Y.F.; Tsai, T.F.; Simon, M.I.; et al. Wolfram Syndrome protein, Miner1, regulates sulphydryl redox status, the unfolded protein response, and Ca ${ }^{2+}$ homeostasis. EMBO Mol. Med. 2013, 5, 904-918. [CrossRef]

51. Dicus, M.M.; Conlan, A.; Nechushtai, R.; Jennings, P.A.; Paddock, M.L.; Britt, R.D.; Stoll, S. Binding of histidine in the (Cys)3(His)1coordinated [2Fe-2S] cluster of human mitoNEET. J. Am. Chem. Soc. 2010, 132, 2037-2049. [CrossRef]

52. Shimomura, Y.; Wada, K.; Fukuyama, K.; Takahashi, Y. The asymmetric trimeric architecture of [2Fe-2S] IscU: Implications for its scaffolding during iron-sulfur cluster biosynthesis. J. Mol. Biol. 2008, 383, 133-143. [CrossRef]

53. Tamir, S.; Paddock, M.L.; Darash-Yahana-Baram, M.; Holt, S.H.; Sohn, Y.S.; Agranat, L.; Michaeli, D.; Stofleth, J.T.; Lipper, C.H.; Morcos, F.; et al. Structure-function analysis of NEET proteins uncovers their role as key regulators of iron and ROS homeostasis in health and disease. Biochim. Biophys. Acta 2015, 1853, 1294-1315. [CrossRef]

54. Wiley, S.E.; Paddock, M.L.; Abresch, E.C.; Gross, L.; van der Geer, P.; Nechushtai, R.; Murphy, A.N.; Jennings, P.A.; Dixon, J.E. The outer mitochondrial membrane protein mitoNEET contains a novel redox-active 2Fe-2S cluster. J. Biol. Chem. 2007, 282, 23745-23749. [CrossRef]

55. Bak, D.W.; Elliott, S.J. Conserved hydrogen bonding networks of MitoNEET tune Fe-S cluster binding and structural stability. Biochemistry 2013, 52, 4687-4696. [CrossRef] [PubMed] 
56. Ferecatu, I.; Gonçalves, S.; Golinelli-Cohen, M.P.; Clémancey, M.; Martelli, A.; Riquier, S.; Guittet, E.; Latour, J.M.; Puccio, H.; Drapier, J.C.; et al. The diabetes drug target MitoNEET governs a novel trafficking pathway to rebuild an Fe-S cluster into cytosolic aconitase/iron regulatory protein 1. J. Biol. Chem. 2014, 289, 28070-28086. [CrossRef] [PubMed]

57. Lill, R. Function and biogenesis of iron-sulphur proteins. Nature 2009, 460, 831-838. [CrossRef] [PubMed]

58. Golinelli-Cohen, M.P.; Lescop, E.; Mons, C.; Gonçalves, S.; Clémancey, M.; Santolini, J.; Guittet, E.; Blondin, G.; Latour, J.M.; Bouton, C. Redox Control of the Human Iron-Sulfur Repair Protein MitoNEET Activity via Its Iron-Sulfur Cluster. J. Biol. Chem. 2016, 291, 7583-7593. [CrossRef] [PubMed]

59. Tan, G.; Liu, D.; Pan, F.; Zhao, J.; Li, T.; Ma, Y.; Shen, B.; Lyu, J. His-87 ligand in mitoNEET is crucial for the transfer of iron sulfur clusters from mitochondria to cytosolic aconitase. Biochem. Biophys. Res. Commun. 2016, 470, 226-232. [CrossRef]

60. Zuris, J.A.; Harir, Y.; Conlan, A.R.; Shvartsman, M.; Michaeli, D.; Tamir, S.; Paddock, M.L.; Onuchic, J.N.; Mittler, R.; Cabantchik, Z.I.; et al. Facile transfer of [2Fe-2S] clusters from the diabetes drug target mitoNEET to an apo-acceptor protein. Proc. Natl. Acad. Sci. USA 2011, 108, 13047-13052. [CrossRef] [PubMed]

61. Eom, K.S.; Cheong, J.S.; Lee, S.J. Structural Analyses of Zinc Finger Domains for Specific Interactions with DNA. J. Microbiol. Biotechnol. 2016, 26, 2019-2029. [CrossRef] [PubMed]

62. Nobili, A.; Krashia, P.; D'Amelio, M. Cisd2: A promising new target in Alzheimer's disease. J. Pathol. 2020, $251,113-116$. [CrossRef] [PubMed]

63. Wang, C.H.; Chen, Y.F.; Wu, C.Y.; Wu, P.C.; Huang, Y.L.; Kao, C.H.; Lin, C.H.; Kao, L.S.; Tsai, T.F.; Wei, Y.H. Cisd2 modulates the differentiation and functioning of adipocytes by regulating intracellular $\mathrm{Ca}^{2+}$ homeostasis. Hum. Mol. Genet. 2014, 23, 4770-4785. [CrossRef]

64. Conlan, A.R.; Axelrod, H.L.; Cohen, A.E.; Abresch, E.C.; Zuris, J.; Yee, D.; Nechushtai, R.; Jennings, P.A.; Paddock, M.L. Crystal structure of Miner1: The redox-active 2Fe-2S protein causative in Wolfram Syndrome 2. J. Mol. Biol. 2009, 392, 143-153. [CrossRef] [PubMed]

65. Shimberg, G.D.; Michalek, J.L.; Oluyadi, A.A.; Rodrigues, A.V.; Zucconi, B.E.; Neu, H.M.; Ghosh, S.; Sureschandra, K.; Wilson, G.M.; Stemmler, T.L.; et al. Cleavage and polyadenylation specificity factor 30: An RNA-binding zinc-finger protein with an unexpected 2Fe-2S cluster. Proc. Natl. Acad. Sci. USA 2016, 113, 4700-4705. [CrossRef]

66. Han, G.; Lu, C.; Guo, J.; Qiao, Z.; Sui, N.; Qiu, N.; Wang, B. C2H2 Zinc Finger Proteins: Master Regulators of Abiotic Stress Responses in Plants. Front. Plant Sci. 2020, 11, 115. [CrossRef]

67. Zhou, X.; Cooper, K.L.; Sun, X.; Liu, K.J.; Hudson, L.G. Selective Sensitization of Zinc Finger Protein Oxidation by Reactive Oxygen Species through Arsenic Binding. J. Biol. Chem. 2015, 290, 18361-18369. [CrossRef]

68. Mozzillo, E.; Delvecchio, M.; Carella, M.; Grandone, E.; Palumbo, P.; Salina, A.; Aloi, C.; Buono, P.; Izzo, A.; D’Annunzio, G.; et al. A novel CISD2 intragenic deletion, optic neuropathy and platelet aggregation defect in Wolfram syndrome type 2. BMC Med. Genet. 2014, 15, 88. [CrossRef]

69. Karmi, O.; Holt, S.H.; Song, L.; Tamir, S.; Luo, Y.; Bai, F.; Adenwalla, A.; Darash-Yahana, M.; Sohn, Y.S.; Jennings, P.A.; et al. Interactions between mitoNEET and NAF-1 in cells. PLoS ONE 2017, 12, e0175796. [CrossRef] [PubMed]

70. Rash-Yahana, M.; Pozniak, Y.; Lu, M.; Sohn, Y.S.; Karmi, O.; Tamir, S.; Bai, F.; Song, L.; Jennings, P.A.; Pikarsky, E.; et al. Breast cancer tumorigenicity is dependent on high expression levels of NAF-1 and the lability of its Fe-S clusters. Proc. Natl. Acad. Sci. USA 2016, 113, 10890-10895. [CrossRef]

71. Chang, N.C.; Nguyen, M.; Germain, M.; Shore, G.C. Antagonism of Beclin 1-dependent autophagy by BCL-2 at the endoplasmic reticulum requires NAF-1. EMBO J. 2010, 29, 606-618. [CrossRef] [PubMed]

72. Chang, N.C.; Nguyen, M.; Shore, G.C. BCL2-CISD2: An ER complex at the nexus of autophagy and calcium homeostasis? Autophagy 2012, 8, 856-857. [CrossRef]

73. Chang, N.C.; Nguyen, M.; Bourdon, J.; Risse, P.A.; Martin, J.; Danialou, G.; Rizzuto, R.; Petrof, B.J.; Shore, G.C. Bcl-2-associated autophagy regulator Naf-1 required for maintenance of skeletal muscle. Hum. Mol. Genet. 2012, 21, 2277-2287. [CrossRef] [PubMed]

74. Kang, R.; Zeh, H.J.; Lotze, M.T.; Tang, D. The Beclin 1 network regulates autophagy and apoptosis. Cell Death Differ. 2011, 18, 571-580. [CrossRef]

75. Chen, Y.F.; Kao, C.H.; Chen, Y.T.; Wang, C.H.; Wu, C.Y.; Tsai, C.Y.; Liu, F.C.; Yang, C.W.; Wei, Y.H.; Hsu, M.T.; et al. Cisd2 deficiency drives premature aging and causes mitochondria-mediated defects in mice. Genes Dev. 2009, 23, 1183-1194. [CrossRef] [PubMed]

76. Rouzier, C.; Moore, D.; Delorme, C.; Lacas-Gervais, S.; Ait-El-Mkadem, S.; Fragaki, K.; Burte, F.; Serre, V.; Bannwarth, S.; Chaussenot, A.; et al. A novel CISD2 mutation associated with a classical Wolfram syndrome phenotype alters $\mathrm{Ca}^{2+}$ homeostasis and ER-mitochondria interactions. Hum. Mol. Genet. 2017, 26, 1599-1611. [CrossRef] [PubMed]

77. Lin, C.C.; Chiang, T.H.; Chen, W.J.; Sun, Y.Y.; Lee, Y.H.; Lin, M.S. CISD2 serves a novel role as a suppressor of nitric oxide signalling and curcumin increases CISD2 expression in spinal cord injuries. Injury 2015, 46, 2341-2350. [CrossRef]

78. Lin, C.C.; Chiang, T.H.; Sun, Y.Y.; Lin, M.S. Protective Effects of CISD2 and Influence of Curcumin on CISD2 Expression in Aged Animals and Inflammatory Cell Model. Nutrients 2019, 11, 700. [CrossRef]

79. Lin, M.S. CISD2 Attenuates Inflammation and Regulates Microglia Polarization in EOC Microglial Cells-As a Potential Therapeutic Target for Neurodegenerative Dementia. Front. Aging Neurosci. 2020, 12, 260. [CrossRef]

80. Kung, W.M.; Lin, C.C.; Kuo, C.Y.; Juin, Y.C.; Wu, P.C.; Lin, M.S. Wild Bitter Melon Exerts Anti-Inflammatory Effects by Upregulating Injury-Attenuated CISD2 Expression following Spinal Cord Injury. Behav. Neurol. 2020, 2020, 1080521. [CrossRef] 
81. Ding, G.; Cheng, L.; Qin, Q.; Frontin, S.; Yang, Q. PPARdelta modulates lipopolysaccharide-induced TNFalpha inflammation signaling in cultured cardiomyocytes. J. Mol. Cell Cardiol. 2006, 40, 821-828. [CrossRef] [PubMed]

82. Galuppo, M.; Di, P.R.; Mazzon, E.; Esposito, E.; Paterniti, I.; Kapoor, A.; Thiemermann, C.; Cuzzocrea, S. GW0742, a high affinity PPAR-b/d agonist reduces lung inflammation induced by bleomycin instillation in mice. Int. J. Immunopathol. Pharmacol. 2010, 23, 1033-1046. [CrossRef] [PubMed]

83. Wu, C.Y.; Chen, Y.F.; Wang, C.H.; Kao, C.H.; Zhuang, H.W.; Chen, C.C.; Chen, L.K.; Kirby, R.; Wei, Y.H.; Tsai, S.F.; et al. A persistent level of Cisd2 extends healthy lifespan and delays aging in mice. Hum. Mol. Genet. 2012, 21, 3956-3968. [CrossRef] [PubMed]

84. Chen, Y.F.; Chou, T.Y.; Lin, I.H.; Chen, C.G.; Kao, C.H.; Huang, G.J.; Chen, L.K.; Wang, P.N.; Lin, C.P.; Tsai, T.F. Upregulation of Cisd2 attenuates Alzheimer's-related neuronal loss in mice. J. Pathol. 2020, 250, 299-311. [CrossRef] [PubMed]

85. Kung, W.M.; Chang, C.J.; Chen, T.Y.; Lin, M.S. Cryogen spray cooling mitigates inflammation and injury-induced CISD2 decline in rat spinal cord hemisection model. J. Integr. Neurosci. 2020, 19, 619-628. [CrossRef] 